Archived version from NCDOCKS Institutional Repository http://libres.uncg.edu/ir/asu/

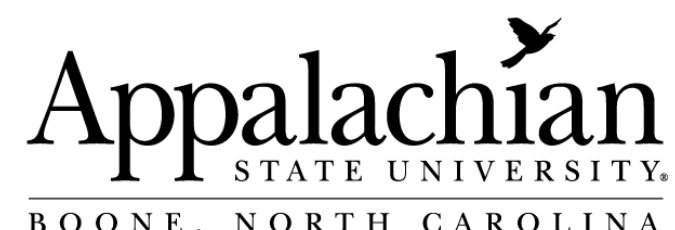

\title{
Immune And Inflammation Responses To A 3-Day Period Of Intensified Running Versus Cycling
}

\author{
By: Dru A. Henson, David C. Nieman, Beibei Luo, Didier Dréau, R. Andrew \\ Shanely, Dustin Dew \& Mary Pat Meaney
}

\begin{abstract}
Functional overreaching has been linked to alterations in immunity and host pathogen defense, but little is known as to whether or not running and cycling evoke different responses. This study compared inflammation, muscle damage and soreness, and innate immune function responses to a 3-day period of intensified exercise in trained long distance runners $(\mathrm{N}=13$, age $34.4+/-2.4$ year $)$ and cyclists $(\mathrm{N}=22$, age $36.6+/-1.7$ year, $\mathrm{P}=0.452)$. Upper respiratory tract infection (URTI) symptomatology was monitored for 12 weeks using the Wisconsin Upper Respiratory Symptom Survey (WURSS), and subjects from both athletic groups came to the lab during week five and exercised $2.5 \mathrm{~h} /$ day for 3 days in a row at 70\% VO2max. Blood samples were collected before and after the 3-day period of exercise, with recovery samples collected 1-, 14-, and $38 \mathrm{~h}$-post-exercise. Samples were analyzed for muscle damage [creatine kinase (CK), myoglobin (MYO)], inflammation (CRP, IL-6, IL-8, IL-10, MCP), and innate immunity [granulocyte and monocyte phagocytosis (GRPHAG and MO-PHAG) and oxidative burst activity (GR-OBA and MO-OBA)]. Runners compared to cyclists experienced significantly more muscle damage (CK 133\% and MYO 404\% higher post-3 days exercise), inflammation (CRP 87\%, IL-6 256\%, IL 8 61\%, IL-10 32\%, MCP 29\%), and delayed onset of muscle soreness (DOMS, 87\%). The 3-day period of exercise caused significant downturns in GR-PHAG, MO-PHAG, GR-OBA, MO-OBA by 14- and 38 h-recovery, but the pattern of change did not differ between groups. No group differences were measured for 12-week URTI severity (18.3 +/- 5.6 and $16.6+/-4.0, \mathrm{P}=0.803)$ and symptom scores $(33.4+/-12.6$ and $24.7+/-5.8, \mathrm{P}=0.477)$. These data indicate that a 3 -day period of functional overreaching results in substantially more muscle damage and soreness, and systemic inflammation in runners compared to cyclists, but without group differences for 12-week URTI symptomatology and post-exercise decrements in innate immune function.
\end{abstract}

Dru A. Henson, David C. Nieman, Beibei Luo, Didier Dréau, R. Andrew Shanely, Dustin Dew \& Mary Pat Meaney (2014) "Immune And Inflammation Responses To A 3-Day Period Of Intensified Running Versus Cycling" Brain, Behavior And Immunity Vol. 39 pp.180-185 [DOI 10.1016/j.bbi.2013.09.004] Version of Record Available From www.researchgate.net 


\section{Introduction}

Most athletes incorporate periodized training in their schedules, with phases of functional overreaching or short-term intensified training to enhance future performance (Meeusen et al., 2013). Single bouts of heavy exertion cause widespread perturbations in physiologic and immune biomarkers, and include transient alterations in innate immune function and elevations in stress hormones, pro-and anti-inflammatory cytokines, and reactive oxygen species (Henson et al, 2008; Nieman, 2001, 2009; Suzulki et al., 2006). These data imply that periods of intensified exercise

training may cause a more serious downturn in immune function and increased incidence of upper respiratory tract infection (URTI), but few well-designed studies have been conducted to verify this hypothesis (Meeusen et al., 2013). Even less is known regarding physiologic and immune responses to functional overreaching in runners compared to cyclists.

Exercise modes incorporating significant eccentric (e.g., running) compared to concentric (e.g., cycling) muscle contractions cause greater muscle damage (Proske and Allen, 2005). The general assumption is that exercise-induced muscle damage is related to alterations in innate immune function and systemic inflammation, but evidence is limited and inconsistent (Paulsen et al., 2012). Bruwnsgaard et al (1997) showed a relationship between elevated creatine kinase (CK) and IL-6 after 30 min of eccentric compared to concentric cycling. However, this has not been a consistent finding, with comparisons across studies difficult to make due to the 
incorporation of a wide variety of exercise challenges and interindividual variation (Paulsen et al., 2012; Peake et al., 2005a,b; Sugama et al., 2012; Suzuki et al., 2006; Toft et al., 2002). At the $160-\mathrm{km}$ Western States Endurance Run (WSER), a strong relationship was found between $\mathrm{CK}$, delayed onset of muscle soreness (DOMS), and a spectrum of systemic inflammation markers including C-reactive protein (CRP), IL-6, granulocyte colony stimulating factor (GC-SF), IL-1 receptor antagonist (IL-1 ra), monocyte chemoattractant-1 (MCP-1), IL-10, and IL-8 (Nieman, 2009; Nieman et al., 2003, 2005, 2006, 2007). During the week following the WSER, DOMS also showed modest positive correlations with many of the cytokines changes experienced during the race (Nieman, 2009). Contrary to expectations, the oxidative stress experienced by the WSER athletes was modest, with no significant correlations to cytokine, CK, and CRP changes (Nieman, 2009; Quindry et al., 2008). Multiple triggers of cytokine release during extreme exercise include elevations in stress hormones and other internal and external stress signals, and metabolic demands such as glycogen deficiency (Muñoz-Cánoves et al., 2013; Pedersen, 2012; Welc and Clanton, 2013).

In a randomized, crossover study of 10 triathletes, single 2.5-h bouts of running and cycling $\left(\sim 75 \% \mathrm{VO}_{2 \max }\right)$ resulted in similar innate immune responses but higher plasma IL-6 concentrations following running (Henson et al., 1999; Nieman et al., 1998a,b). In the current study, these data were extended by comparing muscle damage, inflammation, and innate immune biomarkers in runners and cyclists during a 3-day period of intensified training $(2.5 \mathrm{~h} /$ day), and URTI symptomatology during a 12-week period. We hypothesized that runners compared to cyclists would experience higher muscle damage and inflammation during the 3-day functional overreaching period, with decreased innate immune function and higher overall URTI rates during 12-weeks of monitoring symptoms.

\section{Methods}

\subsection{Subjects and baseline testing}

Subject recruitment was conducted via mass advertising to running and cycling clubs in the Charlotte, NC, metropolitan area. Subjects included healthy, non-smoking runners $(N=13)$ and cyclists $(N=22)$ ages $19-45$ years who regularly competed in race events and were capable of exercising for $2.5 \mathrm{~h} /$ day at high intensity for 3 days in a row in the performance laboratory. All subjects signed informed consent forms, and study procedures were approved by the Institutional Review Board at Appalachian State University. Subjects consented to train normally, maintain weight, and avoid the use of all herbs and medications known to affect inflammation and immune function for the duration of the study (in particular, all non-steroidal anti-inflammatory drugs). Subjects also agreed to avoid use of vitamin and mineral supplements above $100 \%$ the U.S. Daily Value. Prior to the start of the study, runners and cyclists were tested for $\mathrm{VO}_{2 \max }$ using graded exercise tests (with treadmills and cycle ergometers, respectively) with the Cosmed FitMate metabolic device (Rome, Italy). Body composition was measured with the Bod Pod body composition analyzer (Life Measurement, Concord, CA). Demographic and training histories were acquired with questionnaires, and subjects recorded URTI symptoms and minutes trained each day in a log book throughout the entire 12 -week study.

\subsection{Exercise sessions}

Subjects trained normally during the 12-week URTI logging period, and participated in a 3-day period of intensified exercise during week 5. A standardized meal consisting of Boost Plus (Néstle Nutrition, Slorham Park, NJ) was ingested at 12:00 pm each of the 3 days, with energy intake adjusted to $10 \mathrm{kcal} / \mathrm{kg}$ body weight. Boost Plus is a nutritionally complete, high-energy oral supplement with an energy density of $6.4 \mathrm{~kJ} / \mathrm{mL}(1.52 \mathrm{kcal} / \mathrm{mL})$ and $15 \%$ of energy as protein, $35 \%$ as fat, and $50 \%$ as carbohydrate, and 24 vitamins and minerals. Subjects reported to the Human Performance Laboratory at $2: 30 \mathrm{pm}$ and provided blood samples in a rested, seated position. Beginning at 3:00 pm, subjects ran on treadmills or cycled on their own bicycles on CompuTrainers for 2.5 -h at approximately $70 \% \mathrm{VO}_{2 \text { max }}$. Water was given ad libitum throughout the 2.5-h exercise bouts, with no other beverage or food allowed. Heart rate, rating of perceived exertion (RPE), and distance completed were recorded every 30 min during the bout, with oxygen consumption and ventilation measured with the Cosmed FitMate metabolic device after $1 \mathrm{~h}$ of exercise. Subjects repeated this schedule for the next 2 days, but without pre-exercise blood draws. Blood samples were taken immediately following the final exercise bout on the third day, and 14-h- and 38-h-postexercise (i.e., the following two mornings).

\subsection{Complete blood count, creatine kinase, myoglobin, C-reactive protein, DOMS}

Routine complete blood counts were performed by our clinical hematology laboratory using a Coulter Ac.T ${ }^{\mathrm{TM}}$ 5Diff Hematology Analyzer (Beckman Coulter, Inc., Miami, FL) and provided total leukocyte counts, and hemoglobin and hematocrit for the determination of plasma volume change using the method of Dill and Costill (1974). Creatine kinase (CK), myoglobin (MYO), and high-sensitivity C-reactive protein (CRP) were measured using an LX-20 clinical analyzer (Beckman Coulter Electronics, Brea, CA). Delayed onset of soreness (DOMS) was assessed using the 1-10 scale produced by Smith et al. (1993).

\subsection{Plasma cytokines}

Total plasma concentrations of four inflammatory cytokines [monocyte chemoattractant protein 1 (MCP), IL-6, IL-8, and IL-10] were determined using an electrochemiluminescence based solid-phase sandwich immunoassay (Meso Scale Discovery, Gaithersburg, MD, USA). All samples and provided standards were analyzed in duplicate, and the intra-assay CV ranged from $1.7 \%$ to $7.5 \%$ and the inter-assay CV 2.4 to $9.6 \%$ for all cytokines measured. Preand post-exercise samples for the cytokines were analyzed on the same assay plate to decrease inter-kit assay variability.

\subsection{Granulocyte and monocyte phagocytosis (G-PHAG, M-PHAG), oxidative burst activity (G-OBA, M-OBA)}

G-PHAG, M-PHAG, G-OBA, and M-OBA were assayed as previously described (Knab et al., 2013). Briefly, phagocytosis was measured through the uptake of Fluorescein isothiocyanate (FITC)-labeled Staphylococcus aureus bacteria and oxidative burst was measured through the oxidation of non-fluorescent hydroethidine (HE) to fluorescent ethidium bromide in cells stimulated with unlabeled bacteria. Samples were processed on a Q-Prep ${ }^{\mathrm{TM}}$ Workstation (Beckman Coulter, Inc.) and analysis was performed within 18-h of blood collection using a LSR Fortessa flow cytometer (BD Biosciences, San Jose, CA). After gating on the granulocyte and monocyte populations using forward scatter and side scatter (FlowJo analysis software, Tree Star, Inc., Ashland, OR), the mean fluorescence intensity (MFI; $\mathrm{x}$-mean) and percent positive cells for FITC (FL1) and oxidized HE (FL2) were determined. 


\subsection{Upper respiratory tract infection}

The Wisconsin Upper Respiratory Symptom Survey (WURSS) was used to assess URTI severity and symptomatology (Barrett et al., 2009). The WURSS-21 (short version used in this study) includes 10 items assessing symptoms (running nose, plugged nose, sneezing, sore throat, scratchy throat, cough, hoarseness, head congestion, chest congestion, feeling tired), 9 items assessing functional impairments (think clearly, sleep well, breathe easily, walk-climb stairs-exercise, accomplish daily activities, work outside the home, work inside the home, interact with others, live your personal life), and 1 item assessing global severity and global change. Subjects filled in the one-page WURSS-21 at the end of each day during the 12 -week monitoring period. This 12 -week period covered the transition from summer to fall (mid-August to mid-November). From the responses recorded during the 84-day study, an URTI severity score was calculated by summing the daily URTI global severity score $(0=$ not sick, $1=$ very mild URTI to 7 = severe). The URTI symptom score for the 84-day period was calculated by summing all 10 symptom scores for each day's entry $(0=$ do not have this symptom, 1 = very mild to 7 = severe $)$. In similar fashion, the URTI function ability score for the 84-day period was calculated by summing all 9 function scores for each day's entry $(0=$ do not have this symptom, 1 = very mild to $7=$ severe $)$.

\subsection{Statistical procedures}

Data are expressed as mean \pm SE. Subject characteristics and performance data in Table 1 and URTI data in Table 2 were contrasted between groups using independent Student's $t$-tests. All other data were analyzed using a 2 (group) $\times 5$ (time) repeatedmeasures ANOVA, between-groups design. When Box's M suggested that the assumptions necessary for the univariate approach were not tenable, the multivariate approach to repeated measures ANOVA was used (Pillais trace). Changes from pre-exercise to each of four other time points were compared between groups using student's $t$-tests (when interaction effects were significant), with significance set after Bonferroni adjustment $(0.05 / 4)$ at $P \leqslant 0.0125$.

\section{Results}

Subject characteristics and 3-day exercise performance measures are summarized in Table 1. Runners and cyclists were of similar age, body composition, and aerobic capacity, with the runners weighing significantly less than the cyclists. Heart rate, oxygen consumption, and ventilation did not differ significantly between runners and cyclists when comparing averaged data across the three 2.5 -h exercise bouts. The average training volume (minutes per week) during the 12-week monitoring period did not differ between groups (Table 1 ).

The running group included seven males and six females, and the cycling group included 17 males and five females. Male and female athletes (all subjects combined) did not differ in age (36.4 \pm 1.8 and $34.5 \pm 2.1, P=0.557$, respectively), $V_{2 \max }$ $\left(55.7 \pm 1.5\right.$ and $\left.51.1 \pm 2.4 \mathrm{ml} \mathrm{kg}^{-1} \mathrm{~min}^{-1}, P=0.105\right)$, or the percentage of $\mathrm{VO}_{2 \max }$ maintained during the 3-day exercise period (69.4 $\pm 1.6 \%$ and $70.6 \pm 2.6 \%, P=0.701)$. Additionally, a 2 (run, cycle) $\times 2$ (male, female $) \times 5$ (time) repeated measures ANOVA revealed no significant gender effects on all outcome measures shown in Table 2 and Fig. 1 through 3. Thus data comparing runners and cyclists in this paper are presented with male and female athletes combined.

Plasma volume shifts at the end of the third day of exercise did not differ between runners and cyclists $(-1.0 \pm 0.5 \%$ and $-0.8 \pm 0.3 \%, P=0.723$ ). The 3 -day exercise period caused significant increases in CRP, IL-6, IL-8, IL-10, and MCP (time effects, all $P<0.005$ ) (Fig. 1A through 1E). Runners compared to cyclists experienced greater post-3-day-exercise increases in CRP (interaction effect, $P=0.009)$, IL-6 $(P=0.001)$, IL-8 $(<0.001)$, IL-10 $(P=0.045)$, and $\operatorname{MCP}(P<0.001)$. The higher CRP levels in the runners were evident at 38-h post-exercise (Fig. $1 \mathrm{~A}$ ), with no group differences in cytokines measured at 14-h- and 38-h-post-exercise (Fig. 1B through $1 \mathrm{E}$ ). Runners also experienced greater post-3-day-exercise increases in MYO (interaction effect, $P=0.001)$ and $\mathrm{CK}(P<0.001)$ compared to the cyclists (Fig. 2A and B). DOMS ratings were higher in the runners compared to the cyclists immediately- and 1-hpost-3-day exercise, with no differences 14 -h- and 38-h-post-exercise (interaction effect, $P<0.001$ ) (Fig. 3).

Granulocyte and monocyte phagocytosis increased to a similar degree following the 3-day exercise period in both runners and cyclists, and then decreased below pre-exercise levels at 14-h-postexercise (27\% and $28 \%$, respectively; time effect, $P<0.001$ for both; interactions effects $P=0.942$ and $P=0.897$, respectively) (Table 2 ). Granulocyte and monocyte oxidative burst activity also increased in similar fashion following the 3-day exercise period in both runners and cyclists, and then decreased below pre-exercise levels through 38 -h-post-exercise ( $12 \%$ and $21 \%$, respectively; time effect, $P<0.001$ for both; interactions effects $P=0.871$ and $P=0.265$, respectively) (Table 2 ). Total blood leukocyte counts increased in both runners and cyclists, with no group differences in the pattern of change over time (interaction effect, $P=0.176$ ) (data

Table 1

Subject characteristics and 3-day performance measures in runners and cyclists (mean $\pm \mathrm{SE}$ ).

\begin{tabular}{|c|c|c|c|}
\hline Variable, subject characteristics & Runners $(N=13)$ & Cyclists $(N=22)$ & $P$-value \\
\hline Age (yr) & $34.4 \pm 2.4$ & $36.6 \pm 1.7$ & 0.452 \\
\hline Height (m) & $1.70 \pm 0.02$ & $1.75 \pm 0.02$ & 0.090 \\
\hline Weight (kg) & $64.5 \pm 3.0$ & $72.6 \pm 2.39$ & 0.045 \\
\hline Body fat $(\%)$ & $17.3 \pm 1.9$ & $18.8 \pm 1.2$ & 0.492 \\
\hline $\mathrm{VO}_{2 \max }\left(\mathrm{ml} \mathrm{kg}^{-1} \mathrm{~min}^{-1}\right)$ & $54.7 \pm 2.2$ & $54.1 \pm 1.6$ & 0.811 \\
\hline Max heart rate (beats/min) & $179 \pm 2.9$ & $180 \pm 1.9$ & 0.944 \\
\hline 12-wk train volume (min/wk) & $425 \pm 63.6$ & $396 \pm 41.8$ & 0.567 \\
\hline \multicolumn{4}{|c|}{ 3-Day exercise performance measures $(2.5 \mathrm{~h} / \text { day })^{*}$} \\
\hline Beats/min & $149 \pm 3.6$ & $143 \pm 2.7$ & 0.282 \\
\hline$\%$ max heart rate & $82.5 \pm 1.4$ & $79.5 \pm 1.1$ & 0.118 \\
\hline \multicolumn{4}{|l|}{$\mathrm{VO}_{2}$} \\
\hline $\mathrm{ml} \cdot \mathrm{kg}^{-1} \cdot \mathrm{min}^{-1}$ & $39.0 \pm 1.3$ & $36.5 \pm 1.2$ & 0.186 \\
\hline$\% \mathrm{VO}_{2 \max }$ & $71.9 \pm 2.3$ & $68.5 \pm 1.6$ & 0.217 \\
\hline Ventilation (L/min) & $60.7 \pm 3.5$ & $68.5 \pm 3.1$ & 0.111 \\
\hline Rating of perceived exertion & $13.2 \pm 0.2$ & $12.5 \pm 0.3$ & 0.084 \\
\hline
\end{tabular}

Data represent 3-day averages from the three $2.5 \mathrm{~h}$ bouts. 
Table 2

Granulocyte and monocyte phagocytosis and oxidative burst activity (mean $\pm \mathrm{SE}$ ).

\begin{tabular}{|c|c|c|c|}
\hline Variable & Runners $(N=13)$ & Cyclists $(N=22)$ & $P$-value: time effect; interaction effect \\
\hline \multicolumn{4}{|c|}{ Granulocyte phagocytosis (MFI) } \\
\hline Pre-exercise & $7018 \pm 770$ & $7092 \pm 675$ & $<0.001$ \\
\hline Post-3day-exercise & $9901 \pm 620$ & $9393 \pm 676$ & 0.942 \\
\hline $1 \mathrm{~h}$-Post-exercise & $9839 \pm 550$ & $9897 \pm 630$ & \\
\hline 14 h-Post-exercise & $4940 \pm 718$ & $5302 \pm 453$ & \\
\hline 38 h-Post-exercise & $5866 \pm 681$ & $5595 \pm 569$ & \\
\hline \multicolumn{4}{|c|}{ Monocyte phagocytosis (MFI) } \\
\hline Pre-exercise & $5218 \pm 685$ & $4769 \pm 436$ & $<0.001$ \\
\hline Post-3day-exercise & $8343 \pm 454$ & $7806 \pm 652$ & 0.897 \\
\hline 1 h-Post-exercise & $8242 \pm 483$ & $8256 \pm 566$ & \\
\hline 14 h-Post-exercise & $3403 \pm 502$ & $3710 \pm 408$ & \\
\hline 38 h-Post-exercise & $4446 \pm 663$ & $4381 \pm 535$ & \\
\hline \multicolumn{4}{|c|}{ Granulocyte oxidative burst activity (MFI) } \\
\hline Pre-exercise & $8.57 \pm 0.32$ & $8.85 \pm 0.29$ & $<0.001$ \\
\hline Post-3day-exercise & $10.4 \pm 0.38$ & $10.3 \pm 0.49$ & \\
\hline 1 h-Post-exercise & $9.44 \pm 0.93$ & $9.96 \pm 0.62$ & \\
\hline 14 h-Post-exercise & $8.27 \pm 0.44$ & $8.78 \pm 0.57$ & \\
\hline 38 h-Post-exercise & $7.10 \pm 1.07$ & $8.08 \pm 0.77$ & \\
\hline \multicolumn{4}{|c|}{ Monocyte oxidative burst activity (MFI) } \\
\hline Pre-exercise & $5.84 \pm 0.19$ & $5.52 \pm 0.20$ & $<0.001$ \\
\hline Post-3day-exercise & $7.18 \pm 0.29$ & $6.59 \pm 0.32$ & $<0.001$ \\
\hline 1 h-Post-exercise & $6.45 \pm 0.34$ & $6.75 \pm 0.36$ & \\
\hline 14 h-Post-exercise & $5.09 \pm 0.17$ & $4.94 \pm 0.22$ & \\
\hline 38 h-Post-exercise & $4.19 \pm 0.57$ & $4.64 \pm 0.46$ & \\
\hline
\end{tabular}

not shown). Scores for URTI severity, symptoms, and function ability during the 12-week logging period did not differ between the runners and cyclists (Table 3 ).

\section{Discussion}

The 3-day period of intensified exercise was associated with significantly more muscle damage and soreness, and systemic inflammation, in the runners compared to the cyclists, despite similar exercise workload volumes ( $2.5 \mathrm{~h}$ /day). Post-exercise downturns in granulocyte and monocyte phagocytosis and oxidative burst activity, and 12-week URTI symptomatology did not differ between these athletic groups.

This contrast in muscle damage, DOMS, and systemic inflammation during recovery from intensive running (i.e., more eccentric) versus cycling (i.e., more concentric) confirms and extends findings from several other studies. Bruunsgaard et al. (1997) were the first to show higher CK and IL-6 after 30 min of eccentric versus concentric cycling, hypothesizing that this finding was probably related to muscle damage and ensuing inflammatory reactions in the skeletal muscle. However, in a follow-up study utilizing $60 \mathrm{~min}$ of eccentric lower limb exercise, IL-6 increases were modest compared to large CK increases, suggesting that muscle damage was not a major stimulus to IL-6 production, especially when compared to the large IL-6 increases observed during high intensity, long duration cycling (Pedersen, 2012; Toft et al., 2002). The 3-day functional overreaching model utilized in this study showed that under demanding exercise workloads, $7.5 \mathrm{~h}$ of running compared to cycling is associated with significantly more muscle damage (CK was $133 \%$ and MYO 404\% higher post-3 day exercise), muscle soreness (87\% higher), and systemic inflammation (CRP 87\%, IL-6 256\%, IL 8 61\%, IL-10 $32 \%$, and MCP $29 \%$ higher).

Other studies provide variable support for the linkage between muscle damage and systemic inflammation, and part of the problem is that many exercise protocols did not combine the duration and intensity needed to induce sizeable increases in outcome measures (Nieman et al., 2005; Paulsen et al., 2012; Peake et al., 2005a,b; Sugama et al., 2012; Suzuki et al., 2006). Ultramarathon running generates significant muscle damage and soreness, and data collected from 155 athletes competing in the 160-km WSER supported a strong relationship between CK and various cytokines including IL-6 $(r=0.49, P<0.001)$ and GCSF $(r=0.42, P<0.001)$ (Nieman, 2009; Nieman et al., 2005). DOMS was higher the day after the WSER $(7.1 \pm 0.3)$ and was significantly correlated with CK and pre-to-post changes in IL-6, GCSF, IL-10, and MCP (Nieman et al., 2005). Sugama et al. (2012) and Suzuki et al. (2006) also reported concomitant increases in muscle damage and a variety of cytokines during recovery from long endurance, competitive race events.

Welc and Clanton (2013) proposed that IL-6 gene regulation in muscle is responsive to a variety of internal and external stress signals. Although IL-6 can be released from the muscle that has not been significantly damaged from exercise (Fischer, 2006), the highest post-exercise levels reported in the literature are in runners following marathon and ultra-marathon type events that induce muscle damage and DOMS (Nieman, 2001, 2009; Suzuki et al., 2006). In this study, $7.5 \mathrm{~h}$ of running compared to cycling at the same workload intensity over a 3-day period caused more muscle damage and 2.56-fold greater plasma IL-6 concentrations. IL-6 is not only released by the working muscle but targets this tissue, assisting in regulation of muscle satellite cells, energy metabolism, and regeneration (Muñoz-Cánoves et al., 2013). In regenerating muscle, IL-6 is produced by infiltrating neutrophils and macrophages, fibro-adipogenic progenitors, and satellite cells (Joe et al., 2010; Muñoz-Cánoves et al., 2013). Thus there are multiple potential underlying mechanisms linking exercise-induced muscle damage with IL- 6 and other cytokines.

Limited evidence suggests that overreaching is associated with increased incidence of URTI and immune dysfunction, including decreases in neutrophil and monocyte oxidative burst activity, natural killer cell activity, and salivary IgA concentrations, and impaired exercise-induced $\mathrm{CD}^{+} \mathrm{T}$ lymphocyte redistribution (Henson et al., 2008; Lancaster et al., 2004; Meeusen et al., 2013; Morgado et al., 2012; Reid et al., 2004; Robson et al., 1999; Witard et al., 2012). The data from this study support previous findings, with the 3-day period of exercise associated with significant downturns in GR-PHAG ( $\downarrow 27 \%)$, MO-PHAG ( $\downarrow 28 \%)$, GR-OBA $(\downarrow 12 \%)$, and 

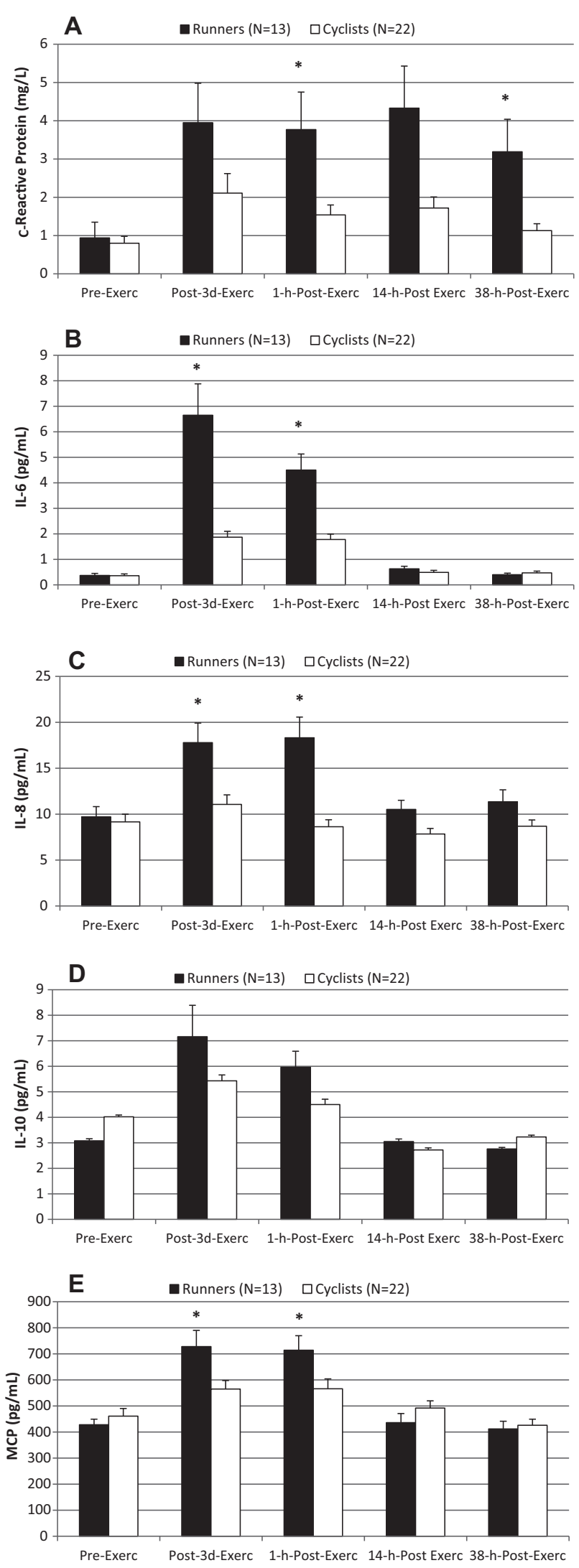

Fig. 1. A through E Serum C-reactive protein (A), plasma IL-6 (B), plasma IL-8 (C), plasma IL-10 (D), and plasma MCP (E) in runners and cyclists before and during $38 \mathrm{~h}$ of recovery from a 3 -day period of intensified exercise (mean $\pm \mathrm{SE}$ ). ${ }^{*} P<0.0125$, between group contrast with pre-exercise.
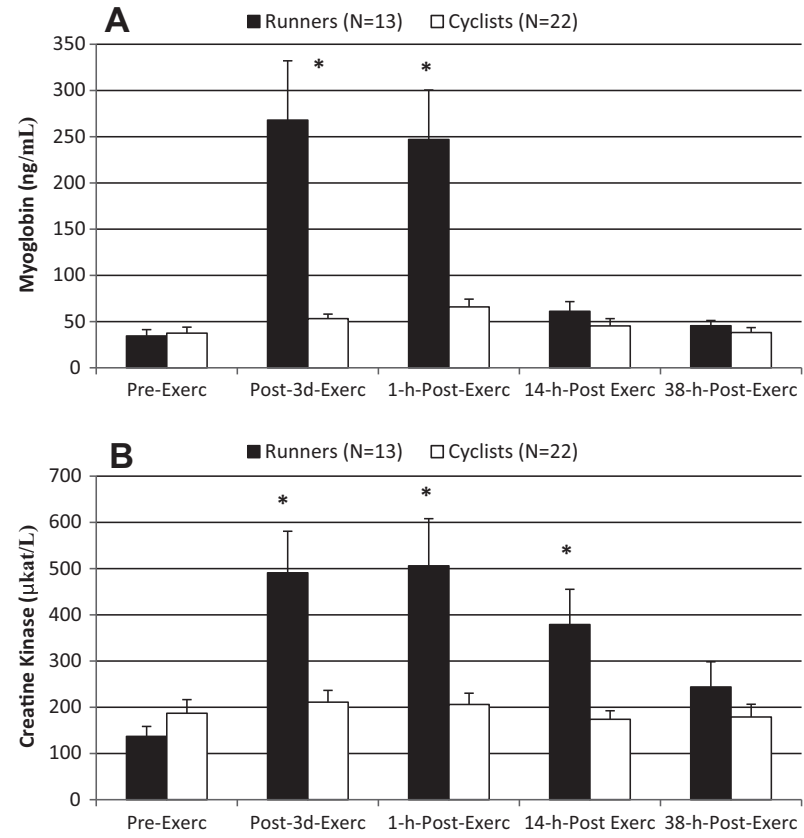

Fig. 2. Serum myoglobin (A) and serum creatine kinase (B) in runners and cyclists before and during $38 \mathrm{~h}$ of recovery from a 3-day period of intensified exercise (mean $\pm \mathrm{SE}$ ). ${ }^{*} P<0.0125$, between group contrast with pre-exercise.

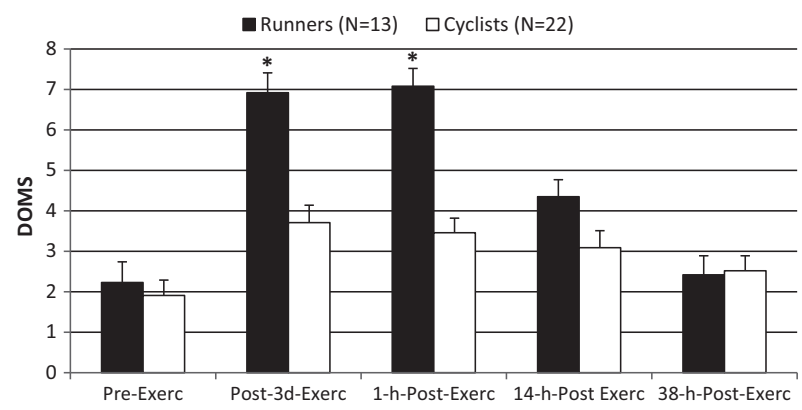

Fig. 3. Delayed onset of muscle soreness (DOMS) in runners and cyclists before and during $38 \mathrm{~h}$ of recovery from a 3 -day period of intensified exercise (mean $\pm \mathrm{SE}$ ). ${ }^{*} P<0.0125$, between group contrast with pre-exercise.

MO-OBA ( $\downarrow 21 \%)$ by 14 - and 38 h-recovery. Downturns in these measures of innate immune function were modest, however, and did not differ between runners and cyclists, despite disparities in muscle damage and systemic inflammation. These results are similar to a previous randomized, crossover study with 10 triathletes who experienced higher IL-6 after running compared to cycling $2.5 \mathrm{~h}$ at $75 \% \mathrm{VO}_{2 \mathrm{max}}$, but similar responses in GR-PHAG, MO-PHAG, GR-OBA, MO-OBA, natural killer cell activity, mitogen-induced lymphocyte proliferation, and stress hormones (Henson et al., 1999; Nieman et al., 1998a,b).

In the current study, no group differences were measured for 12-week URTI severity and symptom scores, and this result is consistent with the lack of group differences for innate immune function (both pre- and post-exercise). Thus despite higher muscle damage, muscle soreness, and inflammation in the runners compared to cyclists after the 3-day period of intensified exercise, there is no indication that this leads to unusual disturbances in innate immune function and or higher 12-week scores for URTI symptomatology. A limitation in the current study is that a sedentary control group was not utilized to allow comparisons in URTI symptoms scores with the athletic groups. 
Table 3

Upper respiratory tract infection (URTI) data (mean \pm SE).

\begin{tabular}{|c|c|c|c|}
\hline Variable & Runners $(N=13)$ & Cyclists $(N=22)$ & $P$-value: interaction effect \\
\hline \multicolumn{4}{|l|}{ URTI measures (12-weeks) } \\
\hline URTI severity score & $18.3 \pm 5.6$ & $16.6 \pm 4.0$ & 0.803 \\
\hline URTI symptom score & $33.4 \pm 12.6$ & $24.7 \pm 5.8$ & 0.477 \\
\hline URTI function ability score & $15.0 \pm 6.4$ & $18.3 \pm 6.0$ & 0.725 \\
\hline
\end{tabular}

In summary, short-term functional overreaching (3 days, $2.5 \mathrm{~h} /$ day, inserted at week 5 during the 12 -week study period) was associated with significantly more muscle damage, DOMS, and systemic inflammation in runners than cyclists. The runners and cyclists engaged in comparable volumes of training over the 12-week study period, and experienced similar scores for URTI symptomatology and 3-day post-exercise downturns in innate immune function. Thus runners compared to cyclists may experience more discomfort during short-term functional overreaching, but without unusual compromises in innate immune function and URTI symptom scores. To limit muscle damage, soreness, and inflammation, runners are encouraged to emphasize periodization in their training schedules, the inclusion of other training modalities, and control of workload volumes to lower levels than can be tolerated by cyclists.

\section{References}

Barrett, B., Brown, R.L., Mundt, M.P., Thomas, G.R., Barlow, S.K., Highstrom, A.D., Bahrainian, M., 2009. Validation of a short form Wisconsin Upper Respiratory Symptom Survey (WURSS-21). Health Qual. Life Outcomes 12 (7), 76.

Bruunsgaard, H., Galbo, H., Halkjaer-Kristensen, J., Johansen, T.L., MacLean, D.A. Pedersen, B.K., 1997. Exercise-induced increase in serum interleukin-6 in humans is related to muscle damage. J. Physiol. 499 (Pt. 3), 833-841.

Dill, D.B., Costill, D.L., 1974. Calculation of percentage changes in volumes of blood, plasma, and red cells in dehydration. J. Appl. Physiol. 37, 247-248.

Fischer, C.P., 2006. Interleukin-6 in acute exercise and training: what is the biological relevance? Exerc. Immunol. Rev. 12, 6-33.

Henson, D.A., Nieman, D.C., Blodgett, A.D., Butterworth, D.E., Utter, A., Davis, J.M., Sonnenfeld, G., Morton, D.S., Fagoaga, O.R., Nehlsen-Cannarella, S.L., 1999. Influence of exercise mode and carbohydrate on the immune response to prolonged exercise. Int. J. Sport Nutr. 9, 213-228.

Henson, D., Nieman, D., Davis, J.M., Dumke, C., Gross, S., Murphy, A., Carmichael, M., Jenkins, D.P., Quindry, J., McAnulty, S., McAnulty, L., Utter, A., Mayer, E., 2008. Post-160-km race illness rates and decreases in granulocyte respiratory burst and salivary IgA output are not countered by quercetin ingestion. Int. J. Sports Med. 29, 856-863.

Joe, A.W., Yi, L., Natarajan, A., Le Grand, F., So, L., Wang, J., Rudnicki, M.A., Rossi, F.M. 2010. Muscle injury activates resident fibro/adipogenic progenitors that facilitate myogenesis. Nat. Cell Biol. 12, 153-163.

Knab, A.M., Nieman, D.C., Gillitt, N.D., Shanely, R.A., Cialdella-Kam, L., Henson, D.A., Sha, W., 2013. Effects of a flavonoid-rich juice on inflammation, oxidative stress, and immunity in elite swimmers: a metabolomics-based approach. Int. J. Sport Nutr. Exerc. Metab. 23, 150-160.

Lancaster, G.I., Halson, S.L., Khan, Q., Drysdale, P., Wallace, F., Jeukendrup, A.E. Drayson, M.T., Gleeson, M., 2004. Effects of acute exhaustive exercise and chronic exercise training on type 1 and type $2 \mathrm{~T}$ lymphocytes. Exerc. Immunol. Rev. 10, 91-106.

Meeusen, R., Duclos, M., Foster, C., Fry, A., Gleeson, M., Nieman, D., Raglin, I., Rietjens, G., Steinacker, J., Urhausen, A., European College of Sport Science American College of Sports Medicine, 2013. Prevention, diagnosis, and treatment of the overtraining syndrome: joint consensus statement of the European College of Sport Science and the American College of Sports Medicine. Med. Sci. Sports Exerc. 45, 186-205.

Morgado, J.M., Rama, L., Silva, I., de Jesus Inácio, M., Henriques, A., Laranjeira, P., Pedreiro, S., Rosado, F., Alves, F., Gleeson, M., Pais, M.L., Paiva, A., Teixeira, A.M. 2012. Cytokine production by monocytes, neutrophils, and dendritic cells is hampered by long-term intensive training in elite swimmers. Eur. I. Appl. Physiol. 112, 471-482.

Muñoz-Cánoves, P., Scheele, C., Pedersen, B.K., Serrano, A.L., 2013. Interleukin-6 myokine signaling in skeletal muscle: a double-edged sword? FEBS I. http:// dx.doi.org/10.1111/febs.12338.
Nieman, D.C., 2009. Immune function responses to ultramarathon race competition. Med. Sportiva 13, 189-196.

Nieman, D.C., Nehlsen-Cannarella, S.L., Fagoaga, O.R., Henson, D.A., Utter, A., Davis, J.M., Williams, F., Butterworth, D.E., 1998a. Effects of mode and carbohydrate on the granulocyte and monocyte response to intensive, prolonged exercise. J. Appl. Physiol. 84, 1252-1259.

Nieman, D.C., Nehlsen-Cannarella, S.L., Fagoaga, O.R., Henson, D.A., Utter, A., Davis, J.M., Williams, F., Butterworth, D.E., 1998b. Influence of mode and carbohydrate on the cytokine response to heavy exertion. Med. Sci. Sports Exerc. 30, 671-678.

Nieman, D.C., Henson, D.A., Smith, L.L., Utter, A.C., Vinci, D.M., Davis, J.M., et al., 2001. Cytokine changes after a marathon race. J. Appl. Physiol. 91, 109-114.

Nieman, D.C., Dumke, C.L., Henson, D.A., McAnulty, S.R., McAnulty, L.S., Lind, R.H., Morrow, J.D., 2003. Immune and oxidative changes during and following the Western States Endurance Run. Int. J. Sports Med. 24, 541-547.

Nieman, D.C., Dumke, C.L., Henson, D.A., McAnulty, S.R., Gross, S.J., Lind, R.H., 2005. Muscle damage is linked to cytokine changes following a $160-\mathrm{km}$ race. Brain Behav. Immun. 19, 398-403.

Nieman, D.C., Henson, D.A., Dumke, C.L., Oley, K., McAnulty, S.R., Davis, J.M., Murphy, E.A., Utter, A.C., Lind, R.H., McAnulty, L.S., Morrow, J.D., 2006. Ibuprofen use, endotoxemia, inflammation, and plasma cytokines during ultramarathon competition. Brain Behav. Immun. 20, 578-584.

Nieman, D.C., Henson, D.A., Davis, J.M., Dumke, C.L., Gross, S.J., Jenkins, D.P., Murphy, E.A., Carmichael, M.D., Quindry, J.C., McAnulty, S.R., McAnulty, L.S., Utter, A.C., Mayer, E.P., 2007. Quercetin ingestion does not alter cytokine changes in athletes competing in the Western States Endurance Run. J. Interferon Cytokine Res. 27, 1003-1011.

Paulsen, G., Mikkelsen, U.R., Raastad, T., Peake, J.M., 2012. Leukocytes, cytokines and satellite cells: what role do they play in muscle damage and regeneration following eccentric exercise? Exerc. Immunol. Rev. 18, 42-97.

Peake, J.M., Suzuki, K., Hordern, M., Wilson, G., Nosaka, K., Coombes, J.S., 2005a. Plasma cytokine changes in relation to exercise intensity and muscle damage. Eur. I. Appl. Physiol. 95, 514-521.

Peake, J.M., Suzuki, K., Wilson, G., Hordern, M., Nosaka, K., Mackinnon, L., Coombes, J.S., 2005b. Exercise-induced muscle damage, plasma cytokines, and markers of neutrophil activation. Med. Sci. Sports Exerc. 37, 737-745.

Pedersen, B.K., 2012. Muscular interleukin-6 and its role as an energy sensor. Med. Sci. Sports Exerc. 44, 392-396.

Proske, U., Allen, T.J., 2005. Damage to skeletal muscle from eccentric exercise. Exerc. Sport Sci. Rev. 33, 98-104.

Quindry, J.C., McAnulty, S.R., Hudson, M.B., Hosick, P., Dumke, C., McAnulty, L.S., Henson, D., Morrow, J.D., Nieman, D., 2008. Oral quercetin supplementation and blood oxidative capacity in response to ultramarathon competition. Int. J. Sport Nutr. Exerc. Metab. 18, 601-616.

Reid, V.L., Gleeson, M., Williams, N., Clancy, R.L., 2004. Clinical investigation of athletes with persistent fatigue and/or recurrent infections. Br. J. Sports Med. $38,42-45$.

Robson, P.J., Blannin, A.K., Walsh, N.P., Castell, L.M., Gleeson, M., 1999. Effects of exercise intensity, duration and recovery on in vitro neutrophil function in male athletes. Int. J. Sports Med. 20, 128-135.

Smith, L.L., Brunetz, M.H., Chenier, T.C., McCammon, M.R., Houmard, J.A., Franklin, M.E., Israel, R.G., 1993. The effects of static and ballistic stretching on delayed onset muscle soreness and creatine kinase. Res. Q. Exerc. Sport. 64, 103-107.

Sugama, K., Suzuki, K., Yoshitani, K., Shiraishi, K., Kometani, T., 2012. IL-17, neutrophil activation and muscle damage following endurance exercise. Exerc. Immunol. Rev. 18, 116-127.

Suzuki, K., Peake, J., Nosaka, K., Okutsu, M., Abbiss, C.R., Surriano, R., Bishop, D., Quod, M.J., Lee, H., Martin, D.T., Laursen, P.B., 2006. Changes in markers of muscle damage, inflammation and HSP70 after an Ironman Triathlon race. Eur. J. Appl. Physiol. 98, 525-534.

Toft, A.D., Jensen, L.B., Bruunsgaard, H., Ibfelt, T., Halkjaer-Kristensen, J., Febbraio, M., Pedersen, B.K., 2002. Cytokine response to eccentric exercise in young and elderly humans. Am. J. Physiol. Cell Physiol. 283, C289-C295.

Welc, S.S., Clanton, T.L., 2013. The regulation of interleukin-6 implicates skeletal muscle as an integrative stress sensor and endocrine organ. Exp. Physiol. 98, 359-371.

Witard, O.C., Turner, J.E., Jackman, S.R., Tipton, K.D., Jeukendrup, A.E., Kies, A.K., Bosch, J.A., 2012. High-intensity training reduces $C D 8^{+}$T-cell redistribution in response to exercise. Med. Sci. Sports Exerc. 44, 1689-1697. 